\title{
Editorial
}

\section{RESEARCH IN SMALL UNIVERSITIES}

\section{J.R.C. PERKIN, President, Acadia University}

Those of us who are faculty members or administrators in small universities in Canada and have been following some of the recent literature about research may be excused if we feel a little apprehensive. For while there are conflicting signs as to what may happen in the larger institutions, there is evidence that research money for smaller places may be increasingly hard to secure.

This should not be taken to imply that the granting agencies are ignorant of, or unsympathetic to, the small universities; indeed, most of the people associated with the administration of research grants seem to realize that we have special concerns.

For example, "Report on the Study of the Research Development Program" prepared by Dr. André Biron and released in June 1985, recognizes and identifies four problems. These may be summarized as:

1. heavy teaching loads

2. lack of equipment, especially in the major equipment category

3. difficulty in obtaining approval for graduate programmes, resulting in a situation in which

4. it is hard to attract highly-qualified faculty.

The report addresses these problems and recommends a developmental initiatives subprogramme especially for small institutions. Awards would be based on slightly modified criteria and would require the elaboration of an approved development plan. There is also a recommendation that a special scheme be worked out for the purchase of equipment costing between $\$ 75,000$ and $\$ 320,000$.

The SSHRC Five-Year Plan also recognizes that small universities face difficulties created by their isolation and the high costs of travel to larger centres, and by the non-availability of specialized library resources. In an attempt to address these problems an enhanced programme of the existing Grants to Small Universities is recommended.

So why should there be apprehension? Perhaps part of an answer is found in looking at other aspects of the same reports and noting the use of such terms as "critical mass," "research centre," "centre of excellence" and "centre of specialization." These terms tend to point in the direction of the larger institutions. For example, far from having a critical mass of scholarship in a given area, most small universities have one or two people for each specialty. Acadia has two anthropologists, three Canadian historians, one plant taxonomist, one specialist in 
the literature of the Atlantic provinces, one expert in international relations, and one philosopher of religion. The problems of transportation and library resources mentioned earlier militate against establishing most kinds of centres of excellence in small universities, although I think there is scope for further thought on this issue.

I think we are seeing another development which makes us uneasy. Several reports bluntly state that in the present financial climate, even with enhanced government support for the granting agencies, there may have to be larger grants and fewer of them if fundamental scientific research is to be encouraged. It is the larger universities which undertake basic research, or at least most of it, and it is in those institutions that the specialists must work. As Arnold Naimark has indicated, we are seeing the emergence of group research. There is good reason for this, but again it produces a picture of which the smaller institutions cannot at present be a part.

The report of the MacDonald Commission contains another theme which stimulates our concern. As we all know, it raises again the topic of a "voucher" system whereby a student would receive all or part of his or her fees to study at a university of his or her choice, to pursue one of the disciplines which are believed to have some national priority. Leaving aside the question of whether such subjects have federal rather than national importance, the voucher scheme might drastically change enrolment patterns at the expense of the small universities. The danger of a new and undesirable kind of competitiveness is obvious, even among the large institutions. So those of us who work in small universities see at best conflicting signs and at worst we see evidence that our capacity for research may be further reduced by the application of policies which, in themselves, seem reasonable and desirable.

But sooner or later some rather frank questions emerge from all these comments about the small and large universities. The question is: Are the small universities worth bothering about? Does not Canada as a whole have too many degreegranting institutions? Would not some academic pruning benefit the Maritimes and perhaps even Ontario as well? Perhaps some really keen competition might be good for the whole system and promote the excellence we talk about so frequently.

These are large questions, and responses rather than answers will be provided here. So let me state a few things about the small universities which I hope will support the assertion that they form an authentic part of the Canadian academic scene, including its research aspect. The points I will make are not exclusively true of the campus with less than 3,000 students but these points ought to be specially true of such places.

1. To begin with the obvious - universities are not research centres, they are teaching institutions. I would quote a brief passage from an impressive little book entitled Excellence in Diversity published in 1983 by the Society for Research into Higher Education - a United Kingdom agency: 
There are some complementarities in both personnel and equipment, which make it convenient for some kinds of research to be undertaken in the same institutions as some kinds of teaching. Obviously the happiest outcome for all occurs when excellent research workers are also excellent teachers. However, good teachers can be poor researchers and vice versa; and teaching and research can compete for the time of staff and for equipment and facilities. (p. 16)

Presumably in a university both emphases will be found, but the emphasis varies from one institution to another.

2. In most small institutions, the honours programme usually involves research and a thesis. So teaching remains basic, but there is an introduction to research, sometimes weeding out those unsuited for it, more often initiating those who will become skilled in it.

3. On a small campus, relationships between teacher and learner may be closer than in a larger context. The student is more easily drawn into the supervisor's research activities and may be given summer employment on a kind of co-operative basis.

4. Because there is usually a core requirement in the degree programme, the student will be forced to see his or her specialization within a wider context which frequently includes study of a second language. I believe this to be an important point and one insufficiently recognized by the MacDonald Commission report.

5. All these lead me to make a dangerously over-simplified distinction between research which is teaching-oriented, and research which is researchoriented. When studied, this distinction may turn out to be as important as the more common distinction between pure and applied research.

At the risk of having my motives misunderstood, I would like to provide a few figures relating to one of Acadia's Departments. I hope that the information may make specific some of the general points made earlier.

The Department in question places great emphasis on its honours programme, although it does offer a master's degree. Every student in the honours programme uses equipment, does research, assists the supervisor, and writes a thesis. In the 53 -year history of the honours programme, 252 students have graduated. Of these, 209 , or $83 \%$ have continued to graduate school or to professional studies in medicine, dentistry or veterinary science. Of the 252 graduates, 131 , or $52 \%$, either already have a doctor's degree or are enrolled in a doctoral programme. If we put together the theses written in the last 53 years by honours students and those from the more recent master's programme, then more than $40 \%$ of the theses have resulted in publications in professional journals. I think this illustrates the value of what I called teaching-oriented research.

The last point I wish to make involves a reference back to some of the reports I have been citing. They all seem to recognize that we need to generate more high-quality graduate students. The SSHRC Five-Year Plan is particularly forthright: 
If nothing is done over the next decade to remedy the situation, Canada will be forced once again to import large numbers of trained and experienced researchers from abroad in the 1990s. (p. 45)

The second NSERC Five-Year Plan also documents the forthcoming shortage of trained personnel. It refers back to its first Five-Year Plan and quotes what was said in 1979:

Even with optimistic assumptions regarding postgraduate university enrollment, it is clear that critical shortages of research talent in the 1980 s will prevent the attainment of the announced goals.

The 1985 Plan adds the comment:

Again we must emphasize that the additional research talent is required even if Canada opts for a policy of greater dependence on imported technology. (p. 128)

To conclude, I would like to make three specific suggestions:

1. If we wish to have researchers in the future, we must have teachers now, men and women who have as their ideal excellence in both teaching and research. To achieve this end, the small universities must be able to secure funds for what I call teaching-oriented research.

2. While I can readily acknowledge that most centres of excellence will be placed in large institutions, some are site-specific. Where a small institution may be the home of a centre without thereby inhibiting its academic excellence, this should be done.

3. While the small universities might only have one person in certain specialties, collectively they may be able to field a team quite as competent as that found in a larger institution. Perhaps this could be seen as a challenge to those who have mastered the mysteries of educational technology. After all, someone did say that this is the age of Telidon rather than Gutenberg!

\section{LA RECHERCHE DANS LES PETITES UNIVERSITES}

\section{J.R.C. Perkin, président, Acadia University}

Il faut comprendre les membres du corps professoral ou les administrateurs de petites universités qui se sentent quelque peu inquiets après avoir lu les dernières publications touchant la recherche. En effet, bien que l'incertitude plane au niveau des institutions plus importantes, il est évident que les subventions de recherche pour les petites universités seront de plus en plus difficiles à obtenir.

Ceci ne signifie pas que les organismes subventionnaires ignorent les petites universités ou ne sympathisent pas à leur cause et, bien sûr, la plupart des gens impliqués dans la gestion des subventions de recherche semblent conscients de nos problèmes particuliers. 
Par exemple, le "Rapport sur l'étude du programme de développement de la recherche" préparé par le Dr Biron et publié en juin 1985, souligne quatre problèmes qui peuvent se résumer ainsi:

1. de lourdes charges d'enseignement

2. un manque d'équipement, principalement dans la catégorie du gros équipement

3. la difficulté d'obtenir l'autorisation d'offrir des programmes d'études supérieures, ce qui mène

4. à l'autre difficulté qui est d'attirer des professeurs hautement qualifiés.

Le rapport étudie ces problèmes et recommande la création d'un programme d'initiatives de développement spécifique aux petites institutions. Les octrois seraient accordés selon des critères légèrement différents et sur présentation d'un plan de développement approuvé. Il recommande également l'élaboration d'un plan spécial pour l'achat d'équipement dont le prix s'échelonne de $75000 \$$ à $320000 \$$.

Le plan quinquennal du CRSH admet également que les petites universités affrontent des difficultés dues à leur isolement, aux coûts du transport vers les grands centres et au manque de ressources documentaires spécialisées. C'est dans le but de régler ces problèmes qu'une révision de l'actuel programme de subventions aux petites universités est recommandée.

Pourquoi tant d'anxiété? La réponse se trouve peut-être, en partie, dans l'étude des autres points de ce rapport où apparaissent des termes tels que "masse critique", "centre de recherche", "centre d'excellence" et "centre de spécialisation", et qui s'adressent plutôt aux grandes institutions. Par exemple, loin d'avoir un nombre important de chercheurs dans un domaine donné, la plupart des petites universités en ont plutôt un ou deux dans chaque discipline. L'Acadia University a deux boursiers en anthropologie, trois en histoire du Canada, un en taxinomie botanique, un spécialiste en littérature des provinces de l'Atlantique, un expert en relations internationales et un en philosophie religieuse. Les problèmes de transport et de ressources documentaires mentionnés plus haut militent contre l'implantation de centres d'excellence dans les petites universités, bien qu'à mon avis il y ait encore des possibilités dans ce domaine.

Je pense que notre malaise vient du fait que nous assistons à un autre développment. Plusieurs rapports affirment carrément que dans la situation financière actuelle, même avec une augmentation de l'aide gouvernementale aux organismes subventionnaires, la recherche scientifique fondamentale devrait être encouragée par des octrois plus importants et moins nombreux. C'est dans les grandes universités, ou du moins dans la plupart d'entre elles, que se fait la recherche fondamentale et là également que les spécialistes doivent travailler. Comme l'a signalé Arnold Naimark, nous assistons à la naissance d'équipes de recherche. Il y a de bonnes raisons pour cela, mais encore une fois, les petites institutions ne disposent pas du cadre adéquat.

Le rapport de la Commission MacDonald aborde un autre chapitre qui nous 
touche également. Comme nous le savons tous, il revient à la charge avec le système de "bons" selon lequel un(e) étudiant(e) recevrait tout ou une partie de ses frais de scolarité pour étudier dans une université de son choix, et dans une des disciplines déclarées "priorité nationale". Mis à part le fait que ces sujets sont d'importance fédérale plutôt que nationale, ce système de bons d'études peut modifier radicalement la répartition des inscriptions aux dépens des petites universités. Le danger d'une nouvelle, et indésirable, sorte de compétitivité est évident, même pour les grandes institutions. Ainsi donc, ceux d'entre nous qui travaillent dans de petites universités voient au mieux des signes contradictoires et au pire la réduction de nos possibilités de recherche en raison de l'application de politiques qui, en elles-mêmes, semblent désirables et raisonnables.

Mais tôt ou tard, de franches questions surgissent de tous ces commentaires au sujet des grandes et des petites universités: Les petites universités valent-elles la peine qu'on s'en occupe? Le Canada, dans son ensemble, n'a-t-il pas trop d'institutions qui décernent des diplômes? Un élagage universitaire ne profiteraitil pas aux Maritimes et peut-être même à l'Ontario? Sans doute, une compétition réellement poussée bénéficierait à l'ensemble du système et aiderait à promouvoir l'excellence dont nous parlons si souvent.

Nous avons là de grandes questions, et nous y répondrons par des réactions plutôt que par des solutions. Permettez-moi de faire quelques remarques sur les petites universités. Remarques qui, j'espère, soutiendront le fait que ces institutions font réellement partie de la scène universitaire et scientifique canadienne. Les affirmations ci-dessous, même si elles s'appliquent aux autres, valent spécialement pour les campus de moins de 3000 étudiants.

1. De toute évidence d'abord, les universités ne sont pas des centres de recherche mais des institutions d'enseignement. Je voudrais mentionner ici un bref passage d'un petit livre impressionnant, intitulé "Excellence in Diversity", publié en 1983 par un organisme britannique, la "Society for Research into Higher Education".

Il y a certaines complémentarités, à la fois dans le personnel et dans l'équipement, qui favorisent certains enseignements et l'entreprise de certains types de recherche dans la même institution. Evidemment, le bonheur le plus parfait règne lorsque d'excellents chercheurs sont aussi d'excellents enseignants. Cependant, de bons professeurs peuvent être de pauvres chercheurs et vice versa; l'enseignement et la recherche peuvent rivaliser lorsqu'il s'agit d'utiliser le temps du personnel, les équipements et les services. (p. 16)

Il est probable que dans une université l'accent sera mis sur les deux aspects mais à des degrés divers selon les institutions.

2. Dans la plupart des petites institutions, les programmes de baccalauréat spécialisé comportent généralement de la recherche et une rédaction de thèse. L'enseignement reste donc fondamental mais il y a une initiation à la recherche, qui élimine parfois ceux qui ne sont pas faits pour cela, et forme ceux qui continueront dans cette voie. 
3. Sur un petit campus, les relations entre le professeur et l'étudiant s'établissent plus aisément que dans un contexte plus vaste. L'étudiant est plus facilement impliqué dans les activités de recherche du directeur de thèse et peut obtenir un emploi d'été basé sur une certaine forme de coopération.

4. Etant donné qu'un programme d'études comporte généralement des cours obligatoires particuliers, l'étudiant devra considérer sa spécialisation dans un contexte élargi qui implique souvent l'étude d'une seconde langue. Je pense qu'il s'agit là d'un aspect important insuffisamment traité dans le rapport de la Commission MacDonald.

5. Toutes ces considérations me conduisent à simplifier à l'extrême la distinction entre la "recherche d'enseignement" et la recherche pure et simple. A l'étude, cette distinction peut s'avérer aussi importante que celle qui est faite plus communément entre la recherche pure et la recherche appliquée.

Au risque d'être incompris, je voudrais fournir quelques données se rapportant à un département de l'Acadia University J'espère que ces informations préciseront les points soulevés plus haut.

Le département en question accorde beaucoup d'importance à ses programmes de baccalauréat spécialisé bien qu'il offre une maîtrise. Chaque étudiant du baccalauréat spécialisé utilise l'équipement; fait de la recherche, assiste le directeur et écrit une thèse. Depuis la création du programme de baccalauréat spécialisé, il y a 53 ans, 252 étudiants ont reçu leur diplôme. De ce nombre, 209, soit $83 \%$, ont poursuivi leurs études dans des écoles supérieures ou dans des secteurs professionnels tels que la médecine, la dentisterie ou la médecine vétérinaire. Des 252 diplômés, 131 , soit $52 \%$, ont déjà un doctorat ou sont inscrits dans un programme de doctorat. Si nous rassemblons les thèses écrites par les étudiants du bac spécialisé pendant ces 53 ans et celles, plus récentes, du programme de maîtrise, nous constatons que $40 \%$ de ces thèses ont fait l'objet de publications dans des revues professionnelles. Je pense que ceci illustre la valeur de ce que j'appelle la recherche-enseignement.

La dernière remarque que j'aimerais faire réfère à quelques-uns des rapports que j'ai cités. Ils semblent tous reconnaître notre besoin de former des étudiants de haut calibre aux études supérieures. Le plan quinquennal du CRSH est particulièrement direct:

Si on ne fait rien dans la prochaine décennie pour remédier à la situation, le Canada sera obligé, une fois encore, de faire appel, dans les années 1990, à un grand nombre de chercheurs étrangers expérimentés. (p. 45)

Le deuxième plan quinquennal du CRSNG démontre aussi le manque flagrant de personnel expérimenté. Il fait référence à son premier plan quinquennal qui stipulait:

Même en étant optimiste à l'égard des inscriptions à l'université au niveau post-doctoral il est clair qu'un manque critique de chercheurs d'élite dans les années 1980 empêchera d'atteindre les buts projetés. 
Le plan de 1985 ajoute:

Nous devons une fois de plus insister sur la nécessité d'une élite plus importante de chercheurs même si le Canada opte pour une politique d'importation de la technologie. (p. 128)

En conclusion, j'aimerais faire trois suggestions spécifiques:

1. Pour l'avenir, si nous souhaitons avoir des chercheurs, nous devons maintenant avoir des enseignants; hommes et femmes ont leurs idéaux d'excellence à la fois dans la recherche et dans l'enseignement. Pour parvenir à cette fin, les petites universités doivent pouvoir obtenir des fonds pour ce que j'appelle la recherche-enseignement.

2. Bien que je puisse déjà prédire que la plupart des centres d'excellence auront leur place dans les grandes institutions, certains de ceux-ci sont spécifiques à l'endroit. Il est possible qu'une petite institution puisse abriter un centre, sans pour cela hypothéquer son excellence académique.

3. Alors que les petites universités peuvent avoir seulement un spécialiste dans un domaine donné, ensemble, elles peuvent être à même de former une équipe aussi compétente que celle que l'on rencontre dans une grande institution. Ceci peut sans doute être perçu comme un défi pour ceux qui ont maîtrisé les mystères de la technologie éducationnelle. Après tout, quelqu'un a déclaré que nous en étions à l'ère de Telidon plutôt qu'à celle de Gutenberg! 